\title{
Level repulsions of bulk acoustic waves in composite materials
}

\author{
Tsung-Tsong $\mathrm{Wu}^{*}$ and Zi-Gui Huang \\ Institute of Applied Mechanics, National Taiwan University, Taipei 106, Taiwan \\ (Received 26 March 2004; revised manuscript received 29 July 2004; published 14 December 2004)
}

\begin{abstract}
Level repulsions of bulk acoustic waves in two-dimensional composite materials and phononic crystals are analyzed in this paper. Detail dispersion curves and displacement fields (polarizations) of bulk acoustic modes in the $\mathbf{k}$ (wave vector) space around the region of level repulsion are calculated and discussed. We show that some cross points of the modes that appear in the dispersion curves in the literature are not true intersections. Those cross points due to the level repulsions in the dispersion curves imply mode anticrossing. The displacement fields of the two modes around the cross points interchange with each other abruptly. On the other hand, the other cross points appearing in the dispersion curves are true intersections which implies that the couplings between the modes are vanishingly small. The continuous displacements of the two wave modes around the cross points provide the continuous dispersion curves. The polarizations of the different modes could be used as the criterion of real or apparent cross points in the dispersion curves.
\end{abstract}

DOI: 10.1103/PhysRevB.70.214304 PACS number(s): 43.40. + s, 43.20. $+\mathrm{g}$, 43.35. $+\mathrm{d}$, 46.40.Cd

Recently, the studies of photonic crystals have led to a rapidly growing interest in the analogous acoustic effects in phononic crystals or periodic elastic structures. Investigations on the band gaps of bulk acoustic waves in composite materials or phononic band structures have been conducted in the past decade. ${ }^{1-10}$ The dispersion relations and the band gap properties of the transverse and mixed polarization modes have been studied, ${ }^{1-3}$ and experimental evidence was given for the existence of absolute acoustic band gaps. ${ }^{4}$ The band gaps for out-of-plane propagation of elastic waves computed in the phononic band structure consisted of an anisotropic infinite square array of parallel quartz rods embedded in an epoxy matrix. ${ }^{5}$

In Refs. 6-9, multiple scattering theory was applied to study the band gaps of bulk wave properties in threedimensional periodic acoustic composites and the band structure of a phononic crystal consisting of complex and frequency-dependent Lame' coefficients. The finite difference time domain method was used to interpret the experimental data of the two-dimensional systems consisting of cylinders of fluids ( $\mathrm{Hg}$, air, and oil) inserted periodically in a finite slab of aluminum host. ${ }^{10}$ Recently, the phononic band gaps of the surface and bulk acoustic modes in twodimensional phononic structures consisting of general anisotropic materials were analyzed. ${ }^{11}$ It is worth noting that there is nothing in the literature that analyzes and discusses the level repulsion effect in two-dimensional composite materials or phononic crystals. Level repulsion which avoids crossing in the distribution of eigenvalues is common knowledge within the physics community. The effect is a phenomenon that is likely to be encountered in any system where there are eigenvalues which depend on some parameter. ${ }^{12-15}$

This paper reports on the occurrence of level repulsion in the dispersion relations of the composite materials and phononic crystals. In this paper, the effects of the level repulsions between the different modes in the dispersion curves of two-dimensional composite materials (PMMA/Ni) and phononic crystals (Ni/PMMA) are analyzed and discussed. The criteria and conditions under which the cross points are distinguished as real or apparent are also dis- cussed. We employed the plane wave expansion method to calculate the dispersion relations and displacement fields of all the bulk acoustic wave (BAW) modes, i.e., the transverse polarization modes [shear vertical (SV) mode with polarization along the filler direction] and mixed polarization modes [shear horizontal $(\mathrm{SH})$ and longitudinal $(L)$ modes with polarization lies in the plane perpendicular to the fillers]. The continuous displacements of the two wave modes around the cross points provide the continuous dispersion curves. The polarizations of the different modes could be used as the criterion of real or apparent cross points in the dispersion curves.

In the following calculations, the formulation based on the plane wave expansion method presented in Ref. 11 was adopted. In an inhomogeneous linear elastic medium with no body force, the equation of motion of the displacement vector $\mathbf{u}(\mathbf{r}, t)$ can be written as

$$
\rho(\mathbf{r}) \ddot{u}_{i}(\mathbf{r}, t)=\partial_{j}\left[C_{i j m n}(\mathbf{r}) \partial_{n} u_{m}(\mathbf{r}, t)\right],
$$

where $\mathbf{r}=(\mathbf{x}, z)=(x, y, z)$ is the position vector and $\rho(\mathbf{r})$ and $C_{i j m n}(\mathbf{r})$ are the position-dependent mass density and elastic stiffness tensor, respectively. We consider a phononic crystal composed of a two-dimensional periodic array ( $x-y$ plane) of material $A$ embedded in a background material $B$. Due to the spatial periodicity, the material constants $\rho(\mathbf{x})$ and $C_{i j m n}(\mathbf{x})$ can be expanded in Fourier series with respect to the twodimensional reciprocal lattice vectors (RLVs), $\mathbf{G}=\left(G_{1}, G_{2}\right)$, as

$$
\begin{gathered}
\rho(\mathbf{x})=\sum_{\mathbf{G}} e^{i \mathbf{G} \cdot \mathbf{x}} \rho_{\mathbf{G}}, \\
C_{i j m n}(\mathbf{x})=\sum_{\mathbf{G}} e^{i \mathbf{G} \cdot \mathbf{x}} C_{\mathbf{G}}^{i j m n},
\end{gathered}
$$

where $\rho_{\mathbf{G}}$ and $C_{\mathbf{G}}^{i j m n}$ are the corresponding Fourier coefficients. 
Dispersion Relations of BAW modes, Cylinder: PMMA / Base: Ni / $f=0.5, R L V=441$

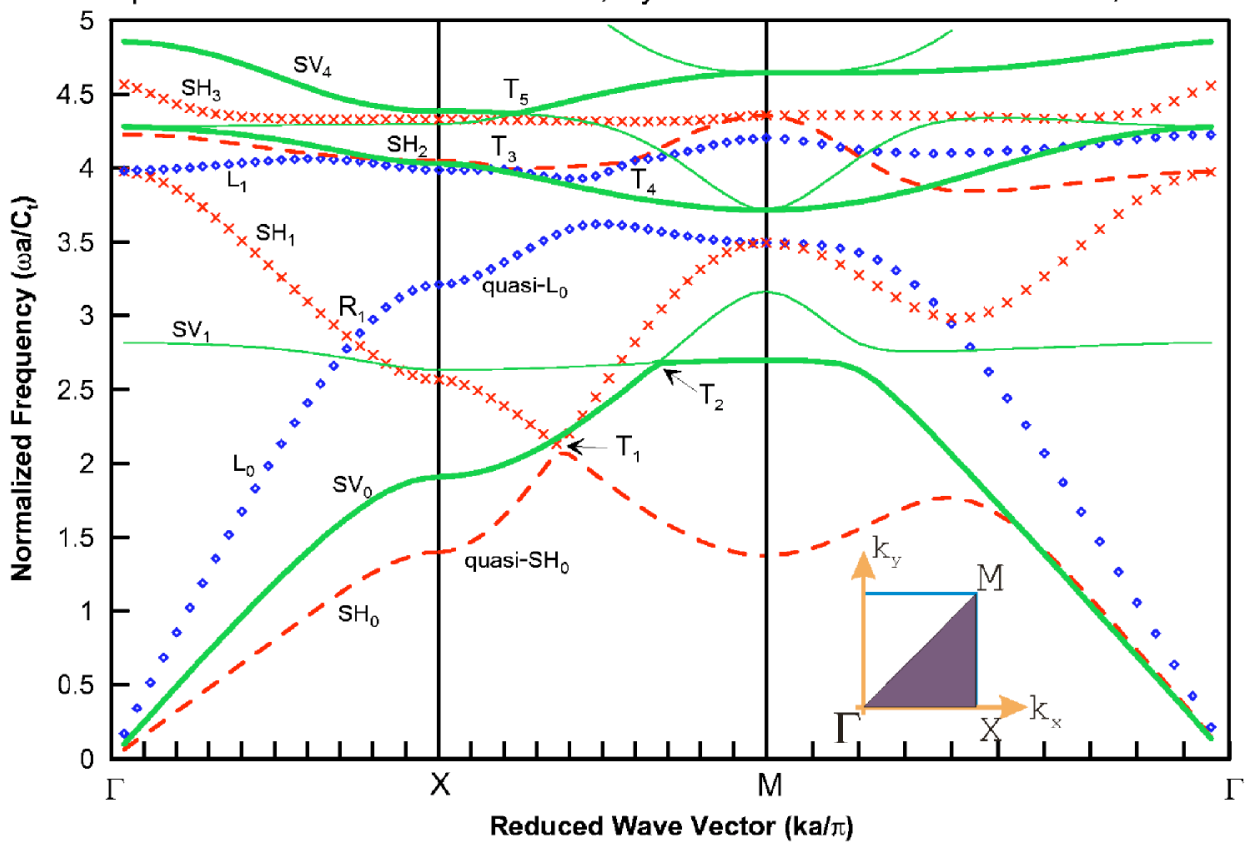

FIG. 1. Dispersion relations of all the bulk modes: pure $\mathrm{SV}, \mathrm{SH}$, and $L$ modes along the $\Gamma-X$ section and the pure SV, quasi-SH, and quasi- $L$ modes along the $X-M$ section in PMMA/Ni composite materials with square lattice. The irreducible part of the Brillouin zone is shown in the inset.

To utilize the Bloch's theorem and to expand the displacement vector $\mathbf{u}(\mathbf{x}, t)$ in Fourier series for bulk wave analysis, we have

$$
\mathbf{u}(\mathbf{x}, t)=\sum_{\mathbf{G}} e^{i \mathbf{k} \cdot \mathbf{x}-i \omega t}\left(e^{i \mathbf{G} \cdot \mathbf{x}} \mathbf{A}_{\mathbf{G}}\right),
$$

where $\mathbf{k}=\left(k_{1}, k_{2}\right)$ is the Bloch wave vector, $\omega$ is the circular frequency, and $\mathbf{A}_{\mathbf{G}}$ is the amplitude of the displacement vector of a bulk acoustic wave.

Substituting Eqs. (2)-(4) into Eq. (1), and after collecting terms systematically, we obtain the eigenvalue problem of bulk waves as

$$
\begin{aligned}
& {\left[\begin{array}{ccc}
M_{\mathbf{G}, \mathbf{G}^{\prime}}^{(1)}-\omega^{2} R_{\mathbf{G}, \mathbf{G}^{\prime}}^{(1)} & L_{\mathbf{G}, \mathbf{G}^{\prime}}^{(1)} & U_{\mathbf{G}, \mathbf{G}^{\prime}}^{(1)} \\
L_{\mathbf{G}, \mathbf{G}^{\prime}}^{(2)} & M_{\mathbf{G}, \mathbf{G}^{\prime}}^{(2)}-\omega^{2} R_{\mathbf{G}, \mathbf{G}^{\prime}}^{(2)} & U_{\mathbf{G}, \mathbf{G}^{\prime}}^{(2)} \\
W_{\mathbf{G}, \mathbf{G}^{\prime}}^{(1)} & W_{\mathbf{G}, \mathbf{G}^{\prime}}^{(2)} & M_{\mathbf{G}, \mathbf{G}^{\prime}}^{(3)}-\omega^{2} R_{\mathbf{G}^{\prime}, \mathbf{G}^{\prime}}^{(3)}
\end{array}\right]} \\
& \times\left[\begin{array}{c}
A_{\mathbf{G}^{\prime}}^{1} \\
A_{\mathbf{G}^{\prime}}^{2} \\
A_{\mathbf{G}^{\prime}}^{3}
\end{array}\right]=0,
\end{aligned}
$$

where the $n \times n$ matrices $M_{\mathbf{G}, \mathbf{G}^{\prime}}^{(1)}, M_{\mathbf{G}, \mathbf{G}^{\prime}}^{(2)}, M_{\mathbf{G}, \mathbf{G}^{\prime}}^{(3)}, L_{\mathbf{G}, \mathbf{G}^{\prime}}^{(1)}, L_{\mathbf{G}, \mathbf{G}^{\prime}}^{(2)}$,

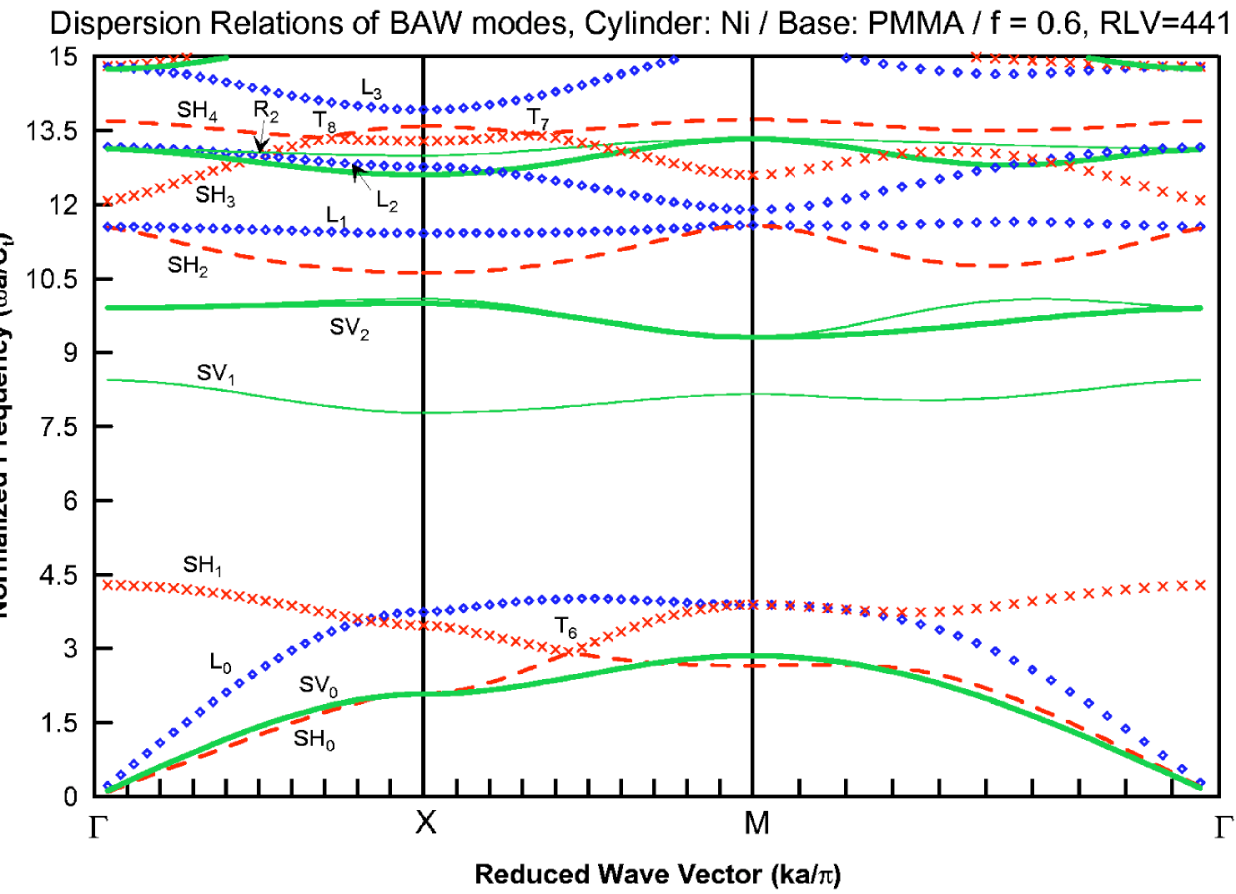

FIG. 2. Dispersion relations of all the bulk modes: pure $\mathrm{SV}, \mathrm{SH}$, and $L$ modes along the $\Gamma-X$ section and the pure SV, quasi-SH, and quasi- $L$ modes along the $X-M$ section in Ni/PMMA phononic band structure with square lattice. 


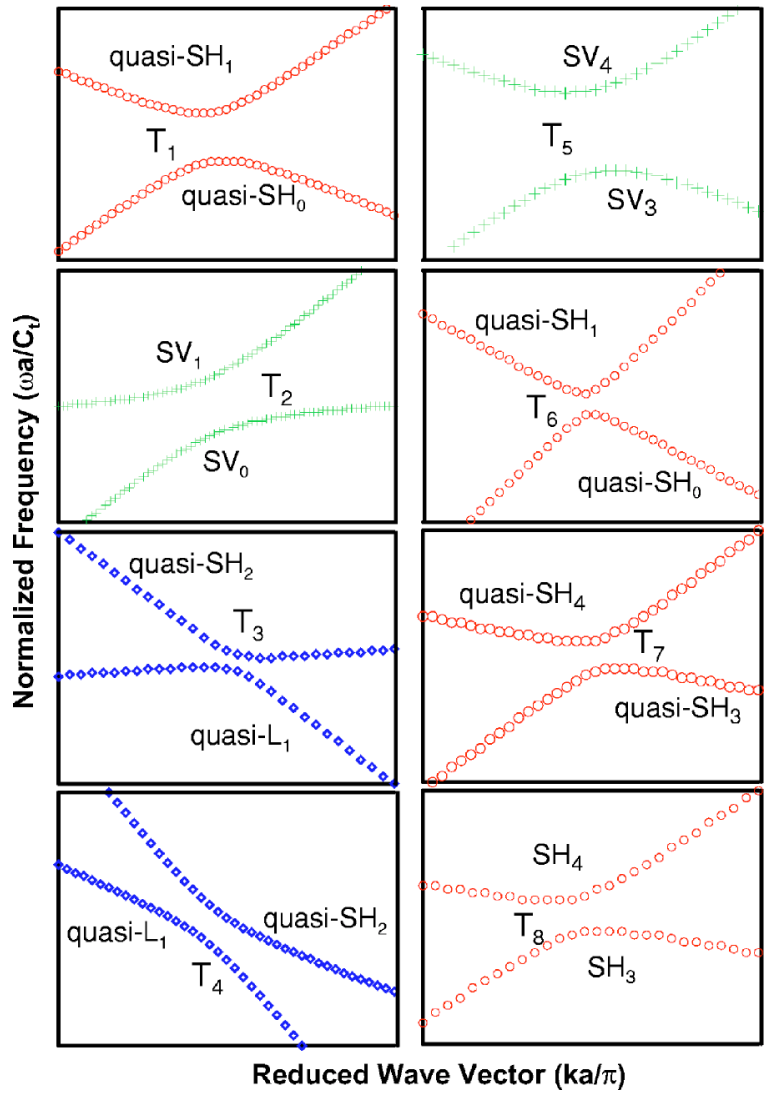

FIG. 3. Enlarge plots of the dispersion curves around the cross points $T_{1}-T_{8}$.

$U_{\mathbf{G}, \mathbf{G}^{\prime}}^{(1)}, U_{\mathbf{G}, \mathbf{G}^{\prime}}^{(2)}, W_{\mathbf{G}, \mathbf{G}^{\prime}}^{(1)}, W_{\mathbf{G}, \mathbf{G}^{\prime}}^{(2)}, R_{\mathbf{G}, \mathbf{G}^{\prime}}^{(1)}, R_{\mathbf{G}, \mathbf{G}^{\prime}}^{(2)}$, and $R_{\mathbf{G}, \mathbf{G}^{\prime}}^{(3)}$ are functions of the Bloch wave vector $\mathbf{k}$, components of the two-dimensional RLV, circular frequency $\omega$, the Fourier coefficients of mass density $\rho_{\mathbf{G}}$, and components of elastic stiffness tensor $C_{\mathbf{G}}^{i j m n} . n$ is the total number of RLVs used in the Fourier expansion and $\mathbf{U}=\left[A_{\mathbf{G}^{\prime}}^{1} A_{\mathbf{G}^{\prime}}^{2} A_{\mathbf{G}^{\prime}}^{3}\right]^{T}$ is the eigenvector used to calculate the displacement fields (polarizations)

$$
\begin{aligned}
& u_{x}=\sum_{\mathbf{G}^{\prime}=1}^{n} e^{i \mathbf{k} \cdot \mathbf{x}-i \omega t}\left(e^{i \mathbf{G}^{\prime} \cdot \mathbf{x}} A_{\mathbf{G}^{\prime}}^{1}\right), \\
& u_{y}=\sum_{\mathbf{G}^{\prime}=1}^{n} e^{i \mathbf{k} \cdot \mathbf{x}-i \omega t}\left(e^{i \mathbf{G}^{\prime} \cdot \mathbf{x}} A_{\mathbf{G}^{\prime}}^{2}\right), \text { and } \\
& u_{z}=\sum_{\mathbf{G}^{\prime}=1}^{n} e^{i \mathbf{k} \cdot \mathbf{x}-i \omega t}\left(e^{i \mathbf{G}^{\prime} \cdot \mathbf{x}} A_{\mathbf{G}^{\prime}}^{3}\right) .
\end{aligned}
$$

It is worth noting that the eigenvalues (resonance frequencies) calculated from Eq. (5) are real by dealing with Hermitian operators, whereas the exceptional points considered in Refs. 12-14 have to do with complex eigenvalues. Equation (5) can be decoupled as the mixed polarization modes [i.e., longitudinal $(L)$ and shear horizontal $(\mathrm{SH})$ ] and shear vertical (SV) modes with polarization of the displacement along the $z$ direction (i.e., the filler's length direction). The explicit formulations of the plane harmonic bulk wave dispersion relations in such a phononic structure in Eq. (5) were listed in Ref. 11.

Consider the PMMA/Ni composite materials and $\mathrm{Ni}$ / PMMA phononic band structures forming the twodimensional square lattices with lattice spacing $a$. Figures 1 and 2 show the dispersion relations of the bulk modes along the boundaries of the irreducible part of the Brillouin zone with filling fractions $f=0.5$ and $f=0.6$ respectively. In the calculations, the $x-y$ plane is parallel to the (001) plane and the $x$ axis is parallel to the [100] direction of Ni and PMMA. The elastic properties of the materials utilized in these two examples are adopted from Ref. 16 and listed in Ref. 17. The vertical axis is the normalized frequency $\omega^{*}=\omega a / C_{t}$ and the horizontal axis is the reduced wave vector $k^{*}=k a / \pi$. $C_{t}$ in Figs. 1 and 2 are the shear velocities of Ni and PMMA for PMMA/Ni and Ni/PMMA band structures, respectively. $k$ is the wave vector along the Brillouin zone. In this paper, we used the 441 numbers of RLV to construct the results in Figs. 1 and 2 and this resulted in a good convergence. In the dispersion relations, the bold solid lines represent the fundamental $\left(\mathrm{SV}_{0}\right)$ and the higher shear vertical modes $\left(\mathrm{SV}_{2}\right.$ and $\mathrm{SV}_{4}$, etc.). The thin solid lines represent the higher shear vertical modes $\left(\mathrm{SV}_{1}\right.$ and $\mathrm{SV}_{3}$, etc.). The square symbols are those for the fundamental $\left(L_{0}\right)$ and the higher longitudinal modes ( $L_{1}$ and $L_{2}$, etc.). The bold dash lines represent the fundamental $\left(\mathrm{SH}_{0}\right)$ and the higher shear horizontal modes $\left(\mathrm{SH}_{2}\right.$ and $\mathrm{SH}_{4}$, etc. $)$ while the lines with $\times$ symbols represent the higher shear horizontal modes $\left(\mathrm{SH}_{1}\right.$ and $\mathrm{SH}_{3}$, etc.).

We note that the $\mathrm{SH}$ and $L$ modes can be decoupled as pure modes in a specific propagation direction and become quasi-SH and quasi- $L$ in the other propagation directions. ${ }^{11,18}$ As the elastic waves propagate along the $x$ axis ( $\Gamma-X$ section), the nonvanishing displacements of the shear horizontal mode, shear vertical mode, and longitudinal mode are $u_{y}, u_{z}$, and $u_{x}$, respectively. For the subsequent modes to appear, we denote them as the fundamental, first, and second modes, etc. For waves propagating along the other directions (i.e., in the $X-M$ sections), they are indeed quasi transverse or longitudinal modes. For convenience, we used the notations of the pure modes at $X$ point to designate the quasi modes in the $X-M$ section.

The calculated results of the dispersion curves in the $X-M$ section shown in Fig. 1 seem to show that the fundamental and the higher shear horizontal modes, quasi- $\mathrm{SH}_{0}$ and quasi- $\mathrm{SH}_{1}$, cross over at point $T_{1}$; the dispersion curves of the higher shear horizontal modes and the higher longitudinal modes (quasi-SH $\mathrm{SH}_{2}$ and quasi- $L_{1}$ ) crossover at points $T_{3}$ and $T_{4}$. The dispersion curves of the shear vertical modes and higher shear vertical modes $\left(\mathrm{SV}_{0}\right.$ and $\mathrm{SV}_{1}, \mathrm{SV}_{3}$ and $\left.\mathrm{SV}_{4}\right)$ make crosses at points $T_{2}$ and $T_{5}$ in the $X-M$ section. In Fig. 2 , the dispersion curves of the fundamental and the higher shear horizontal modes, quasi- $\mathrm{SH}_{0}$ and quasi- $\mathrm{SH}_{1}$, cross over at point $T_{6}$; the dispersion curves of the two higher shear horizontal modes (quasi- $\mathrm{SH}_{3}$ and quasi- $\mathrm{SH}_{4}$ ) crossover at points $T_{7}$. It is worth noting that the dispersion curves of the two higher shear horizontal modes in the $\Gamma-X$ section $\left(\mathrm{SH}_{3}\right.$ and $\mathrm{SH}_{4}$ ) cross over at points $T_{8}$. However, by decreasing the values of both of the frequency and the wave vector intervals in the calculations around the points of $T_{1}-T_{8}$ (Fig. 3), we 


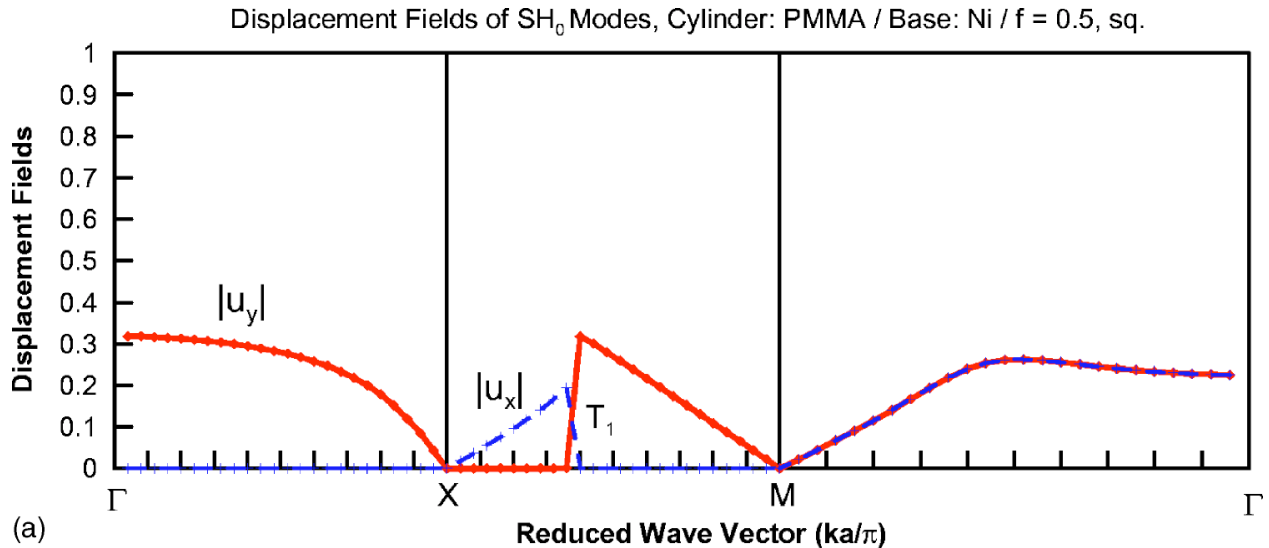

(a)
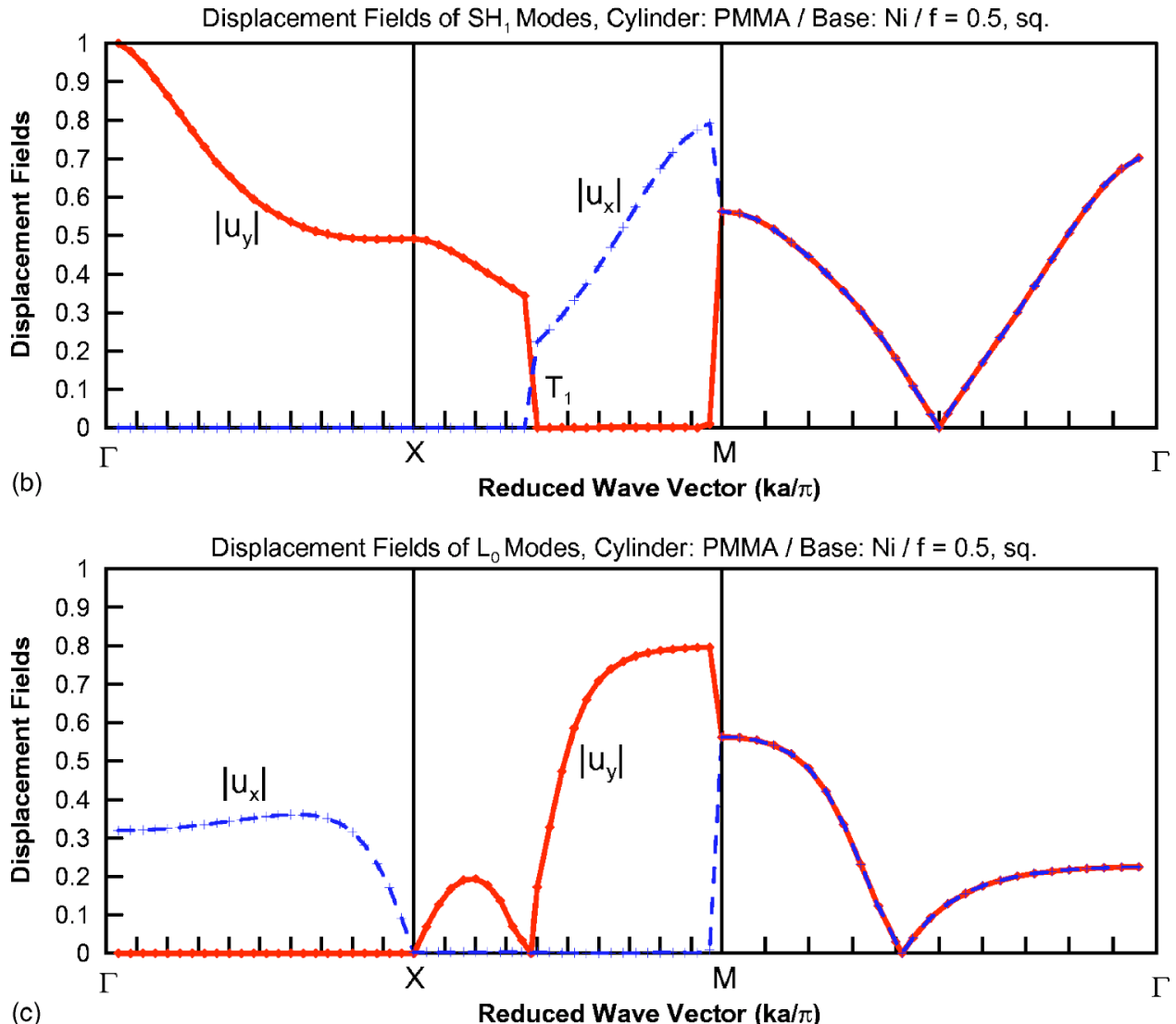

FIG. 4. Displacement fields of (a) $\mathrm{SH}_{0}$ (b) $\mathrm{SH}_{1}$ (c) $L_{0}$ modes along the irreducible part of the Brillouin zone. found that the intersections of the modes mentioned above are not true intersection, instead, these modes repel away from each other due to the level repulsions at these points.

To further examine the characteristics of the level repulsions at these points, we calculated the displacement fields of the $\mathrm{SH}_{0}, \mathrm{SH}_{1}$, and $L_{0}$ modes in PMMA/Ni band structure along all the boundaries of the irreducible part of Brillouin zone shown in Figs. 4(a)-4(c), respectively. Along the $\Gamma-X$ section, the nonvanishing displacement of the shear horizontal mode is $u_{y}$ as expected and it vanishes at the band gap point $X$ in Fig. 4(a). As the propagating direction rotates away from the $X$ point, one finds that $u_{y}$ remains very small; however, $u_{x}$ increases gradually until point $T_{1}$. At this particular propagating direction, $u_{x}$ suddenly jumps to a very small value, while $u_{y}$ jumps from a small value to a finite value and then decays to a small value at the band gap point
$M$. It is worth noting that in the $\mathrm{SH}_{0}$ mode, the magnitudes of the displacements $u_{x}$ and $u_{y}$ in the $\Gamma-M$ section are equal due to the symmetry of the lattice arrangements. The relative amplitudes of the displacements $\left|u_{x}\right|$ and $\left|u_{y}\right|$ shown in Fig. 4 are normalized to the maximum value of $\left|u_{y}\right|$ of the $\mathrm{SH}_{1}$ modes in the $\Gamma-X$ section [Fig. 4(b)].

Similar to the displacement fields of the fundamental shear horizontal mode shown in Fig. 4(a), Figs. 4(b) and 4(c) show the calculated results of the displacement fields of the $\mathrm{SH}_{1}$ and $L_{0}$ modes. From Fig. 4, we found clearly that the level repulsion phenomena appear at the sharp bend of the dispersion curves. Through detailed calculations, we found the similar results occurring at points $T_{3}$ and $T_{4}$ in Fig. 1 and points $T_{6}, T_{7}$, and $T_{8}$ in Fig. 2 . It is worth noting that level repulsion not only exists in the mixed modes $(\mathrm{SH}, L)$ but also in the decoupled SV modes. For example, level repulsion 


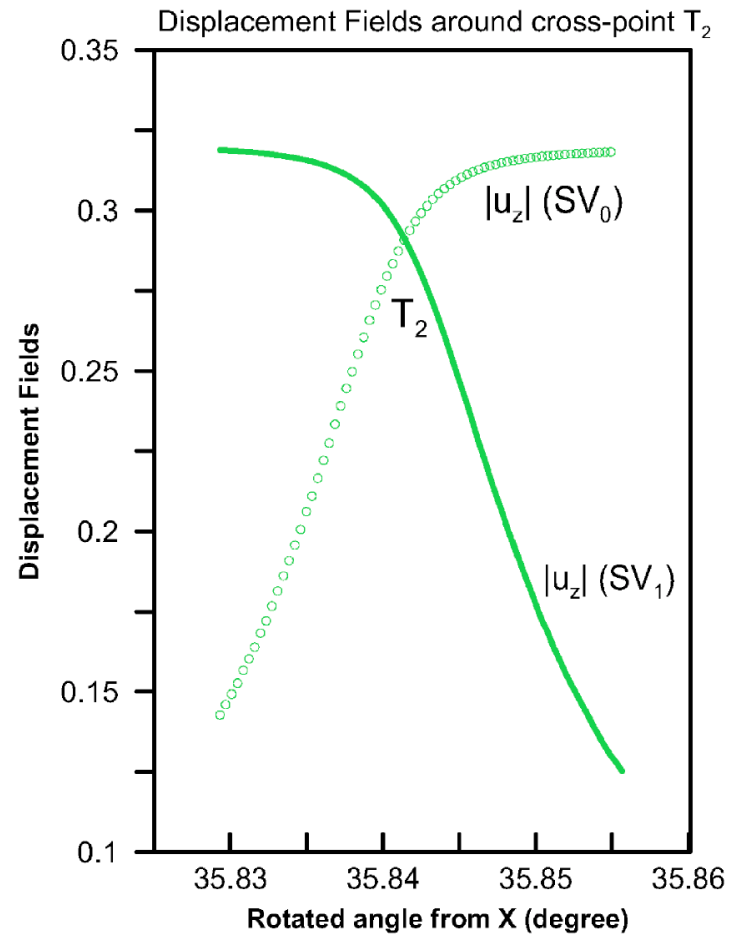

FIG. 5. Displacement fields around the cross point $T_{2}$.

phenomena occur between $\mathrm{SV}_{0}$ and $\mathrm{SV}_{1}$ modes $\left(T_{2}\right), \mathrm{SV}_{3}$ and $\mathrm{SV}_{4}$ modes $\left(T_{5}\right)$ in the $X-M$ section of Fig. 1. Figure 5 shows the displacement fields around point $T_{2}$. The vertical axis is the displacement responses and the horizontal axis is the angle rotated from the $X$ point. In Fig. 5, around the point $T_{2}$, we observe that the phenomenon and the displacement responses jump rapidly and interchange with each other. From the results shown above, we conclude that in the dispersion curves, the displacement fields change rapidly and interchange with each other around those points where level repulsions occurred.

On the other hand, we note that the other cross points in the dispersion curves of Figs. 1 and 2 are real, i.e., the dispersion curves are true intersections. The reason for causing the true intersections of the dispersion curves is due to the vanishing small mode couplings. To further demonstrate that the cross points appeared in the dispersion curves of Figs. 1 and 2 are whether real or apparent, we calculated the displacement fields of the different modes around the cross points $R_{1}$ [Fig. 6(a)] and $T_{1}$ [Fig. 6(b)] in the $\Gamma-X$ and $X-M$ sections of Fig. 1 . The cross point $R_{1}$ represents the intersection of the $L_{0}$ mode and $\mathrm{SH}_{1}$ mode with propagating direction along the $x$ axis. As shown in Fig. 6(a), the nonvanishing displacement of the $L_{0}$ mode $\left(u_{x}\right)$ and the $\mathrm{SH}_{1}$ mode $\left(u_{y}\right)$ are continuous through the $\Gamma-X$ section and therefore it is confirmed as a real cross point; that is, there is no coupling between these two modes. Figure 6(b) shows the apparent cross-point in the $X-M$ section of Fig. 1. Two sharp changes of $u_{x}$ and $u_{y}$ between the quasi-SH $\mathrm{SH}_{1}$ and quasi- $\mathrm{SH}_{0}$ modes are found. We note that as the propagation direction turning away from the $X$ point, those SH modes are not pure SH modes; instead, they are quasi transverse mode and coupled with the longitudinal mode.

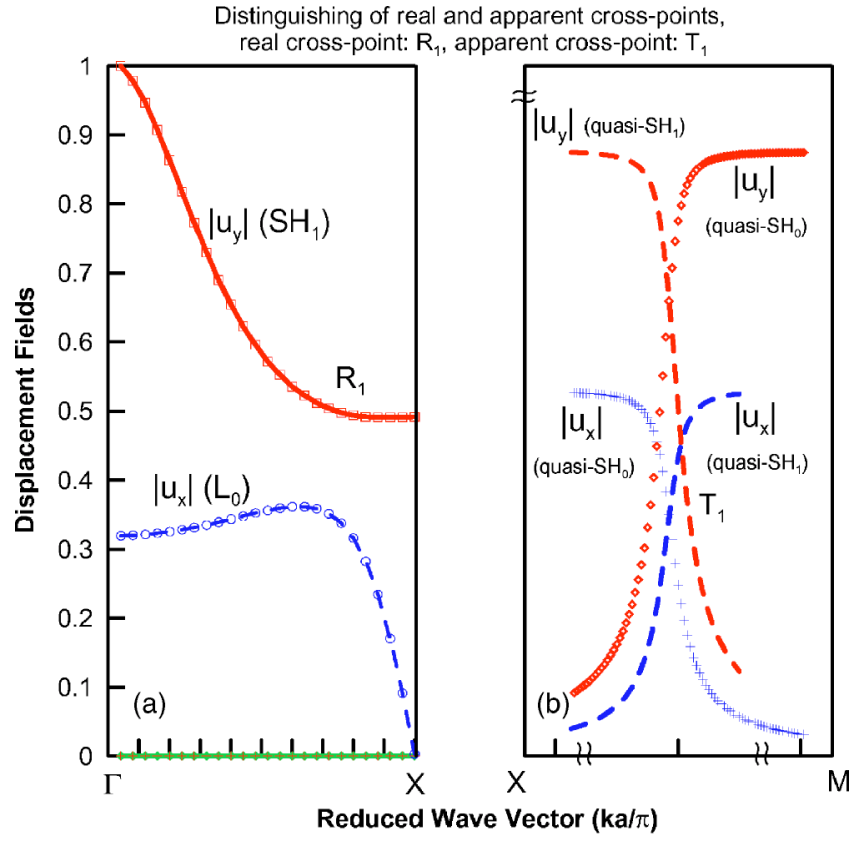

FIG. 6. Real and apparent cross points: real cross point $R_{1}$ and apparent cross point $T_{1}$.

The ultranarrow band gaps induced by the level repulsion in composite materials or phononic crystals may be sorted into two groups: Group I $\left(T_{2}, T_{3}\right.$, and $\left.T_{4}\right)$ and group II $\left(T_{1}\right.$, $T_{5}, T_{6}, T_{7}$, and $\left.T_{8}\right)$. The bends of the dispersion curves in group I are smooth, no frequency gap exists between the two dispersion curves. In group II, the sharp bends of the dispersion curves induce a very small frequency band gap width. For example, the width of the band gap around the $T_{1}$ point is $0.000026 \omega^{*}$ and located rotated $20.35^{\circ}$ from the $X$ point.

In summary, we have examined the level repulsion phenomena of BAW modes in two-dimensional composite materials and phononic band structures. From the detail calculations of the dispersion curves and the displacement fields of BAW modes in the $\mathbf{k}$ space, we observed the level repulsion and the peculiar effects on the displacement fields of the modes. We found that the cross points of the modes appeared in the dispersion curves of Figs. 1 and 2 are apparent due to the strong mode couplings. The dispersion curves around the apparent cross points, in fact, approach to each other and then bend away follow the trace of the opposite mode. Furthermore, the displacements of the two modes around the cross points interchange with each other abruptly. On the other hand, the other cross points appeared in the dispersion curves of Figs. 1 and 2 are real and in fact they are due to the vanishing small mode couplings. The continuous displacements of the two wave modes around the cross points provide the continuous dispersion curves. Finally, the polarizations of the different modes due to the level repulsions could be used as the criterion of real or apparent cross-points in the dispersion curves.

The authors thank the National Science Council (Grant No. NSC92-2212-E-002-058) and the NTU-ITRI center of Taiwan for financial support. 
*Email address: wutt@ ndt.iam.ntu.edu.tw

${ }^{1}$ M. S. Kushwaha, P. Halevi, L. Dobrzynski, and B. DjafariRouhani, Phys. Rev. Lett. 71, 2022 (1993).

${ }^{2}$ M. S. Kushwaha, P. Halevi, G. Martinez, L. Dobrzynski, and B. Djafari-Rouhani, Phys. Rev. B 49, 2313 (1994).

${ }^{3}$ J. O. Vasseur, B. Djafari-Rouhani, L. Dobrzynski, M. S. Kushwaha, and P. Halevi, J. Phys.: Condens. Matter 6, 8759 (1994).

${ }^{4}$ J. O. Vasseur, P. A. Deymier, G. Frantziskonis, G. Hong, B. Djafari-Rouhani, and L. Dobrzynski, J. Phys.: Condens. Matter 10, 6051 (1998).

${ }^{5}$ M. Wilm, A. Khelif, S. Ballandras, and V. Laude, Phys. Rev. E 67, 065602 (2003).

${ }^{6}$ M. Kafesaki and E. N. Economou, Phys. Rev. B 60, 11993 (1999).

${ }^{7}$ I. E. Psarobas and N. Stefanou, Phys. Rev. B 62, 278 (2000).

${ }^{8}$ Zhengyou Liu, C. T. Chan, and Ping Sheng, Phys. Rev. B 62, 2446 (2000).

${ }^{9}$ Jun Mei, Zhengyou Liu, Jing Shi, and Decheng Tian, Phys. Rev. B 67, 245107 (2003).
${ }^{10}$ D. Garica-Pablos, M. Sigalas, F. R. Montero de Espinosa, M. Kafesaki, and N. Garcia, Phys. Rev. Lett. 84, 4349 (2000).

${ }^{11}$ Tsung-Tsong Wu, Zi-Gui Huang, and S. Lin, Phys. Rev. B 69, 094301 (2004).

${ }^{12}$ W. D. Heiss, Phys. Rev. E 61, 929 (2000).

${ }^{13}$ C. Dembowski, H.-D. Graf, H. L. Harney, A. Heine, W. D. Heiss, H. Rehfeld, and A. Richter, Phys. Rev. Lett. 86, 787 (2001).

${ }^{14}$ M. Philipp, P. von Brentano, G. Pascovici, and A. Richter, Phys. Rev. E 62, 1922 (2000).

${ }^{15}$ Volker Ahlers, R. Zillmer, and Arkady Pikovsky, Phys. Rev. E 63, 036213 (2001).

${ }^{16}$ B. A. Auld, Acoustic Fields and Waves in Solids, 2nd ed. (Kreiger, Malabar, FL, 1990).

${ }^{17}$ We use the elastic constants $C_{11}=7.11$ and $C_{44}=2.03$ (in units of GPa) and mass density $\rho=1200 \mathrm{~kg} / \mathrm{m}^{3}$ for PMMA, and $C_{11}$ $=32.4$ and $C_{44}=8.0$ (in units of $10^{10} \mathrm{~Pa}$ ) and mass density $\rho$ $=8905 \mathrm{~kg} / \mathrm{m}^{3}$ for nickel.

${ }^{18}$ Daniel Royer and Eugene Dieulesaint, Elastic Waves in Solids I: Free and Guided Propagation (Springer-Verlag, Berlin, 2000). 